\title{
miR-653-5p suppresses the growth and migration of breast cancer cells by targeting MAPK6
}

\author{
MEI ZHANG ${ }^{1}$, HONGWEI WANG ${ }^{2}$, XIAOMEI ZHANG ${ }^{3}$ and FENGPING LIU ${ }^{2}$ \\ Departments of ${ }^{1}$ Radiotherapy Technology, ${ }^{2}$ Operating Theatre and ${ }^{3}$ Gynaecology, \\ Linyi Cancer Hospital, Linyi, Shandong 276000, P.R China
}

Received August 2, 2019; Accepted June 15, 2020

DOI: $10.3892 / \mathrm{mmr} .2021 .11839$

\begin{abstract}
Breast cancer is the worldwide leading cause of cancer-related deaths among women. Increasing evidence has demonstrated that microRNAs (miRNAs) play critical roles in the carcinogenesis and progression of breast cancer. miR-653-5p was previously reported to be involved in cell proliferation and apoptosis. However, the role of miR-653-5p in the progression of breast cancer has not been studied. In the present study, it was found that overexpression of miR-653-5p significantly inhibited the proliferation, migration and invasion of breast cancer cells in vitro. Moreover, overexpression of miR-653-5p promoted cell apoptosis in breast cancer by regulating the $\mathrm{Bcl}-2 / \mathrm{Bax}$ axis and caspase- 9 activation. Additionally, the epithelial-mesenchymal transition and activation of the Akt/mammalian target of rapamycin signaling pathway were also inhibited by miR-653-5p. Furthermore, the data demonstrated that miR-653-5p directly targeted mitogen-activated protein kinase 6 (MAPK6) and negatively regulated its expression in breast cancer cells. Upregulation of MAPK6 could overcome the inhibitory effects of miR-653-5p on cell proliferation and migration in breast cancer. In conclusion, this study suggested that miR-653-5p functions as a tumor suppressor by targeting MAPK6 in the progression of breast cancer, and it may be a potential target for breast cancer therapy.
\end{abstract}

\section{Introduction}

Breast cancer, which is one of the most prevalent and aggressive malignancies, is the leading cause of cancer-related deaths among women worldwide $(1,2)$. Tumor metastasis is associated with the high mortality rates in patients with breast cancer (3). Despite significant advances in the systematic treatment of breast cancer, which has significantly improved the prognosis

Correspondence to: Dr Fengping Liu, Department of Operating Theatre, Linyi Cancer Hospital, 6 Lingyuan East Street, Lanshan, Linyi, Shandong 276000, P.R China

E-mail: 1123794383@qq.com

Key words: breast cancer, miR-653-5p, mitogen-activated protein kinase 6 , proliferation, migration rates of patients, the treatment effect on patients with metastatic breast cancer remains unsatisfactory (4). Additionally, chemotherapy resistance is also a major issue in the treatment of patients with breast cancer (5). Therefore, the molecular mechanisms of tumorigenesis and development of breast cancer should be further explored, and new therapeutic targets identified to develop a novel strategy for breast cancer treatment.

It has been demonstrated that the occurrence and progression of breast cancer are complex multi-step processes, which involves genetic alterations and epigenetic modifications (6). Accumulating evidence has demonstrated that microRNAs (miRNAs), a class of small non-coding RNAs, can either promote the degradation of mRNA or inhibit the translation of the target gene by binding to its 3'untraslated region (UTR), thereby regulating the expression of the target gene at the post-transcriptional level $(7,8)$. Previous studies have revealed that some miRNAs are dysregulated in various cancers and it has been proposed that they have key roles in carcinogenesis and tumor progression by regulating the expression of oncogenes or tumor suppressor genes $(9,10)$. Recently, several studies have demonstrated that miRNAs play critical roles in the progression of breast cancer, and may even function as promising biomarkers or prognostic markers (11-13). miR-653, located at chromosome 7q21.3, has been previously reported to be involved in the proliferation and apoptosis of mice thymocytes by targeting tripartite motif containing 9 (14). Furthermore, Yuan et al (15) demonstrated that miR-653 regulates cell proliferation, invasion and apoptosis in non-small cell lung cancer. However, the role of miR-653-5p in the progression of breast cancer are yet to be elucidated.

In the present study, the biological role of miR-653-5p in the progression of breast cancer and the underlying mechanism were investigated. Herein, the data demonstrated that miR-653-5p mimics inhibited the proliferation, migration and invasion of breast cancer cells, as well as promoting cell apoptosis, suggesting the suppressive role of miR-653-5p in breast cancer. Moreover, investigation into the underlying mechanism showed that miR-653-5p acted as a tumor suppressor in breast cancer cells by targeting mitogen-activated protein kinase 6 (MAPK6).

\section{Materials and methods}

Cell culture and transfection. Human breast cancer cell lines MCF-7 and MDA-MB-231 were obtained from The Cell 
Bank of Type Culture Collection of the Chinese Academy of Sciences and cultured in RPMI-1640 medium (HyClone; GE Healthcare Life Sciences) supplemented with 10\% FBS (Gibco; Thermo Fisher Scientific, Inc.), penicillin (100 U/ml; Sigma-Aldrich; Merck KGaA) and streptomycin $(0.1 \mathrm{mg} / \mathrm{ml}$; Sigma-Aldrich; Merck $\mathrm{KGaA}$ ) at $37^{\circ} \mathrm{C}$ with $5 \% \mathrm{CO}_{2}$.

miR-653-5p mimics (5'-GUGUUGAAACAAUCUCUA CUG-3') and miRNA negative control (miR-NC; 5'-UUU GGUAAAAUUCAACCAGCUA-3') were synthesized by Guangzhou RiboBio Co., Ltd.. The pcDNA3.1-MAPK6 plasmid was constructed by Guangzhou RiboBio Co., Ltd., the blank pcDNA3.1 vector was used as control. Cells $\left(2 \times 10^{5}\right.$ cells/well $)$ were plated into 6-well plates and incubated at $37^{\circ} \mathrm{C}$ with $5 \% \mathrm{CO}_{2}$ overnight. At 40-60\% confluence, MCF-7 and MDA-MB-231 cells were transfected with miR-653-5p mimics $(50 \mathrm{nM})$, miR-NC (50 $\mathrm{nM})$, pcDNA3.1-MAPK6 $(5 \mu \mathrm{g})$ and pcDNA3.1 $(5 \mu \mathrm{g})$ using Lipofectamine $2000^{\circledast}$ (Invitrogen; Thermo Fisher Scientific, Inc.), following the manufacturer's protocols.

Reverse transcription-quantitative $P C R \quad(R T-q P C R)$ analysis. Total RNA extracted from transfected MCF-7 and MDA-MB-231 cells with an Ultrapure RNA kit (CWBio) were reverse transcribed into cDNA at $42^{\circ} \mathrm{C}$ for 50 min and $85^{\circ} \mathrm{C}$ for 5 min with the miRNA cDNA Synthesis kit (CWBio), following the manufacturer's manual. RT-qPCR analysis was then performed following the instructions of the miRNA qPCR Assay kit (CWBio). The following thermocycling conditions were used for qPCR: Initial denaturation at $95^{\circ} \mathrm{C}$ for $10 \mathrm{~min}$; followed by 40 cycles of $95^{\circ} \mathrm{C}$ for $15 \mathrm{sec}$ and $60^{\circ} \mathrm{C}$ for $1 \mathrm{~min}$; and final extension at $72^{\circ} \mathrm{C}$ for $50 \mathrm{sec}$. miR-653-5p and MAPK6 expression levels were calculated using the $2^{-\Delta \Delta C q}$ method (16) and normalized to the internal reference genes U6 and GAPDH, respectively. The following primers were used for qPCR: miR-653-5p forward, 5'-GTGTTGAAACAA TCTCTACTG-3' and reverse, 5'-GAACATGTCTGCGTA TCTC-3'; U6 forward, 5'-CTCGCTTCGGCAGCACA-3' and reverse, 5'-ACGCTTCACGAATTTGCGTGTC-3'; MAPK6 forward, 5'-AGCGCTAGAGGAAGCATCAC-3' and reverse, 5'-GTGGGATGCCTATGGACTCG-3'; and GAPDH forward, 5'-TATGATGATATCAAGAGGGTAGT-3' and reverse, 5'-TGTATCCAAACTCATTGTCATAC-3'.

Cell counting kit-8 (CCK-8) assay. Following $24 \mathrm{~h}$ of transfection, $\sim 1 \times 10^{3}$ breast cancer cells were plated into a 96-well plate and cultured at $37^{\circ} \mathrm{C}$. CCK-8 reagent (10 $\mu \mathrm{l}$; Beijing Solarbio Science \& Technology Co., Ltd.) was added into each well of the plate at $0,24,48$ and $72 \mathrm{~h}$, according to the manufacturer's instructions. Following incubation at $37^{\circ} \mathrm{C}$ for $1.5 \mathrm{~h}$, the absorbance at $450 \mathrm{~nm}$ was measured with a microplate reader.

Colony formation assay. Following transfection for $24 \mathrm{~h}$, cells were collected and digested with $0.25 \%$ trypsin. Cells were seeded into $60 \mathrm{~mm}$ plates with a density of $5 \times 10^{2}$ cells/plate and cultured for 1-2 weeks. After visible colonies were formed, cells were fixed in $4 \%$ paraformaldehyde at room temperature for 30 min then stained with $0.1 \%$ crystal violet stain at room temperature for $30 \mathrm{~min}$. Finally, the number of colonies was counted. Colonies were defined as the cell population ( $>50$ cells) of the descendants of $>6$ generations of a single proliferating cell in vitro.
Transwell assay. Transwell chambers (EMD Millipore) with or without Matrigel (BD Biosciences) for $1 \mathrm{~h}$ at $37^{\circ} \mathrm{C}$ in 24-well plates were used to assess cell migration and invasion in vitro. Briefly, following transfection for $24 \mathrm{~h}$, cells were suspended in serum-free medium, then $1 \times 10^{5}$ cells were added into the top chamber. RPMI-1640 medium (500 $\mu 1)$ supplemented with $20 \%$ FBS was used as a chemoattractant and added into the bottom chamber. Following incubation for $24 \mathrm{~h}$, the migrating or invading cells were fixed in $4 \%$ paraformaldehyde for $30 \mathrm{~min}$ at room temperature then stained with $0.1 \%$ crystal violet for another $20 \mathrm{~min}$ at room temperature. Stained cells were captured and counted in nine randomly selected fields of view under a light microscope (magnification, x100). The number of migratory or invasive cells was quantified using ImageJ (version 6.0; National Institutes of Health).

Flow cytometry. Breast cancer cells transfected with miR-653-5p mimics or miR-NC for $24 \mathrm{~h}$ were collected and cultured in serum-free medium for another $24 \mathrm{~h}$. After cells were digested with trypsin and centrifuged at $999 \times \mathrm{g}$ for $5 \mathrm{~min}$ at $4^{\circ} \mathrm{C}$, they were suspended with buffer solution and the cell density was adjusted to $1-5 \times 10^{6}$ cells $/ \mathrm{ml}$. Cell suspension $(100 \mu \mathrm{l})$ was stained with Annexin V/FITC and PI (BioVision, Inc.) at room temperature for $5 \mathrm{~min}$ in the dark. The proportion of apoptotic cells was analyzed by flow cytometry (BD FACSCanto II; BD Biosciences) and FlowJo software (version 7.6.5; FlowJo LLC). The proportion of apoptotic cells was calculated as the sum of early (Annexin V/FITC+/PI-) and late (Annexin V/FITC+/PI+) apoptotic cells.

Western blot analysis. After $48 \mathrm{~h}$ of transfection, total protein was extracted from the breast cancer cells using RIPA lysis buffer (CWBio) supplemented with protease inhibitor cocktail (CWBio) and quantified using a bicinchoninic acid assay kit (Beyotime Institute of Biotechnology). Total protein $(20 \mu \mathrm{g})$ for each group were loaded on a $10 \%$ gel, resolved using SDS-PAGE and subsequently transferred to the PVDF membrane (EMD Millipore). The membranes were blocked with 5\% skimmed milk for $1 \mathrm{~h}$ at room temperature and incubated overnight at $4^{\circ} \mathrm{C}$ with the following primary antibodies (Cell Signaling Technology, Inc.): Anti-E-cadherin (1:1,000; cat. no. 14472), anti-N-cadherin (1:1,000; cat. no. 4061), anti-Vimentin (1:1,000; cat. no. 49636), anti-Bcl-2 (1:1,000; cat. no. 15071), anti-Bax (1:1,000; cat. no. 2772), anti-cleaved Caspase 3 (1:1,000; cat. no. 9661), anti-Akt (1:1,000; cat. no. 9272), anti-p-Akt (1:1,000; Ser473; cat. no. 4060), anti-mTOR (1:1,000; cat. no. 2972), anti-p-mTOR (1:1,000; Ser2448; cat. no. 2971), anti-MAPK6 (1:1,000; cat. no. 4067) and anti-GAPDH (1:1,000; cat. no. 2118). The blots were incubated with the horseradish peroxidase-conjugated secondary antibodies (1:3,000; cat. no. SA00001-2; ProteinTech Group, Inc.) for $1 \mathrm{~h}$ at room temperature and visualized using an ECL reagent (CWBio). Signals were analyzed with Quantity One software (version 4.6.6; Bio-Rad Laboratories, Inc.).

Dual-luciferase reporter assay. TargetScan (version 7.2; www.targetscan.org/vert_72) and StarBase (version 2.0; starbase.sysu.edu.cn/starbase2/index.php) were used to predict the potential target gene of miR-653-5p. The wild-type $(w t)$ or 

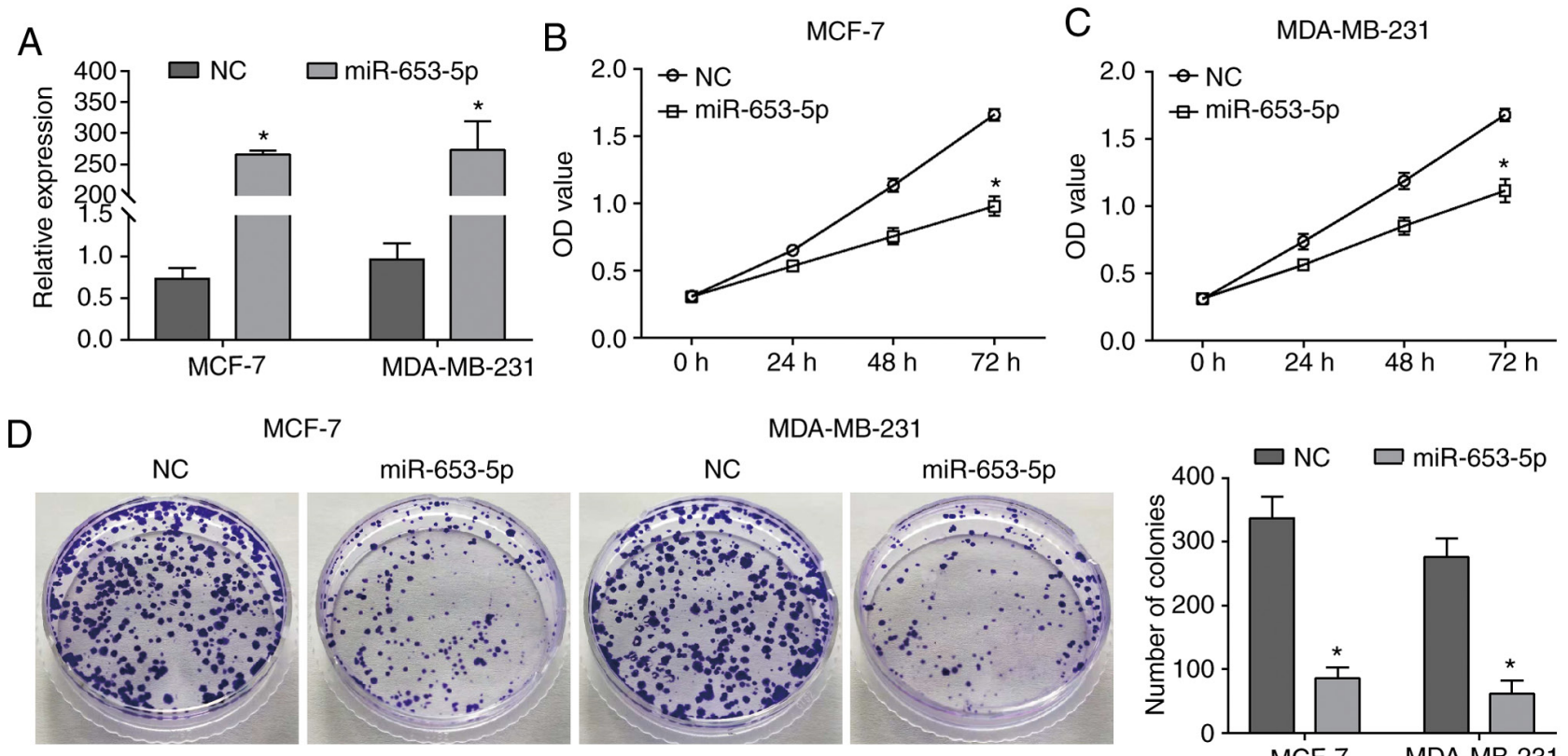
MCF-7
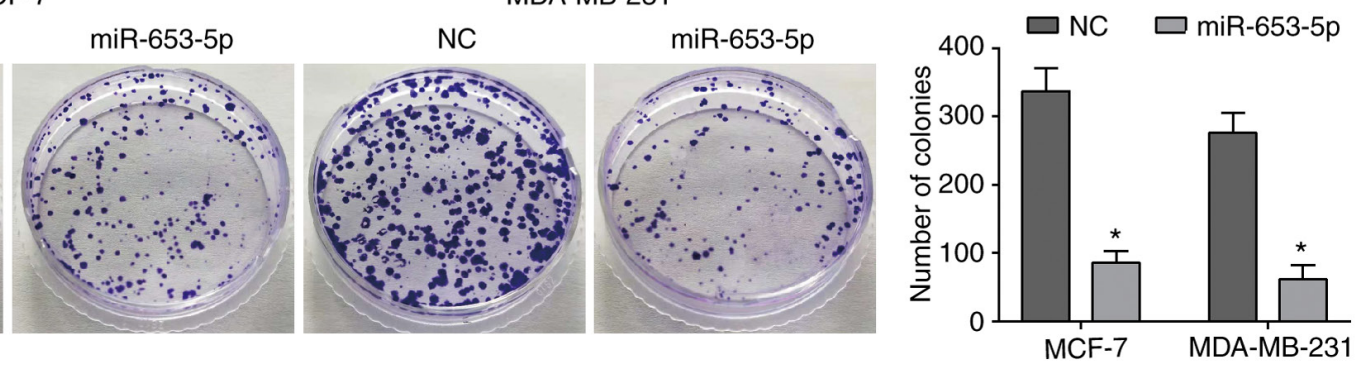

Figure 1. miR-653-5p inhibits the proliferation of breast cancer cells. (A) Expression of miR-653-5p in breast cancer cells MCF-7 and MDA-MB-231 transfected with miR-653-5p mimics. The viability of (B) MCF-7 and (C) MDA-MB-231 cells transfected with miR-653-5p mimics was determined using a Cell Counting Kit-8 assay. (D) Colony formation abilities of MCF-7 and MDA-MB-231 cells were assessed using colony-formation assay. Experiments were performed in triplicate. ${ }^{*} \mathrm{P}<0.05$ vs. NC. NC, negative control; miR, microRNA.

mutant-type (mut) of the 3'UTR of MAPK6 was cloned into the pmirGLO vector (Promega Corporation). 293 T cells $\left(1 \times 10^{6}\right)$ were co-transfected with pmirGLO-MAPK6-wt/-mut plasmid $(1 \mu \mathrm{g})$ and $50 \mathrm{nM}$ miR-653-5p mimics or miR-NC using Lipofectamine $2000^{\circledR}$ (Invitrogen; Thermo Fisher Scientific, Inc.). Following incubation for $48 \mathrm{~h}$, cells were collected and luciferase activity was measured using the Dual-luciferase Report Assay system (Promega Corporation). Firefly luciferase activity was normalized to Renilla luciferase activity.

Statistical analysis. Data are expressed as the mean \pm SD of three independent repeats. An unpaired Student's t-test was used for comparison between two groups, and one-way ANOVA followed by Tukey's post hoc test was used for multiple comparisons. All statistical analyses were performed using GraphPad Prism software 7.0 (GraphPad Software, Inc.). $\mathrm{P}<0.05$ was considered to indicate a statistically significant difference.

\section{Results}

Upregulation of miR-653-5p inhibits the proliferation of breast cancer cells. To investigate the function of miR-653-5p in breast cancer, MCF-7 and MDA-MB-231 cells were transfected with miR-653-5p mimics to upregulate the expression of miR-653-5p (Fig. 1A). As indicated by the CCK-8 assay, upregulation of miR-653-5p caused a significant decrease in cell viability compared with the $\mathrm{NC}$ group (Fig. 1B and C). The suppressive effects of miR-653-5p on cell proliferation was further demonstrated by a significant decrease in the number of colonies in the miR-653-5p overexpression group compared with the NC group (Fig. 1D). Therefore, these results suggested that miR-653-5p functions as a suppressor in the proliferation of breast cancer cells.
Upregulation of miR-653-5p reduces cell migration, invasion and epithelial-mesenchymal transition (EMT) in breast cancer cells. The effect of miR-653-5p on cell migration was assessed using a Transwell assay. As shown in Fig. 2A, overexpression of miR-653-5p significantly reduced the migratory activity of both MCF-7 and MDA-MB-231 cells compared with the control group. Additionally, cells transfected with miR-653-5p mimics displayed a significant decrease in the number of cells that invaded through the Matrigel-coated membrane (Fig. 2A). As commonly known, EMT is a critical event in the initiation of the metastatic cascade and contributes to the metastasis of cancer cells. Therefore, further study was performed to assess the effect of miR-653-5p on the process of EMT in breast cancer cells. The data demonstrated that the expression of epithelial marker E-cadherin was significantly upregulated by miR-653-5p, whereas a significant decrease in the expression of mesenchymal markers, N-cadherin and vimentin, was observed in cells transfected with miR-653-5p mimics (Fig. 2B), suggesting that miR-653-5p suppresses EMT in breast cancer cells. Collectively, these data indicated that the inhibitory effect of miR-653-5p on cell invasion may be related to its ability to suppress EMT in breast cancer cells.

miR-653-5p induces cell apoptosis and inhibits the Akt/mammalian target of rapamycin (mTOR) signaling pathway in breast cancer cells. The effect of miR-653-5p on cell apoptosis in breast cancer cells was investigated using flow cytometry. As indicated in Fig. 3A, miR-653-5p mimics increased the apoptotic rate of MCF-7 and MDA-MB-231 cells compared with the control group. To further investigate the underlying mechanism of increased apoptosis, western blot analysis was performed to evaluate the expression of apoptosis-related proteins. It was demonstrated that, compared with the NC group, miR-653-5p overexpression downregulated 
A

MCF-7

MDA-MB-231 NC $\square \operatorname{miR}-653-5 p$
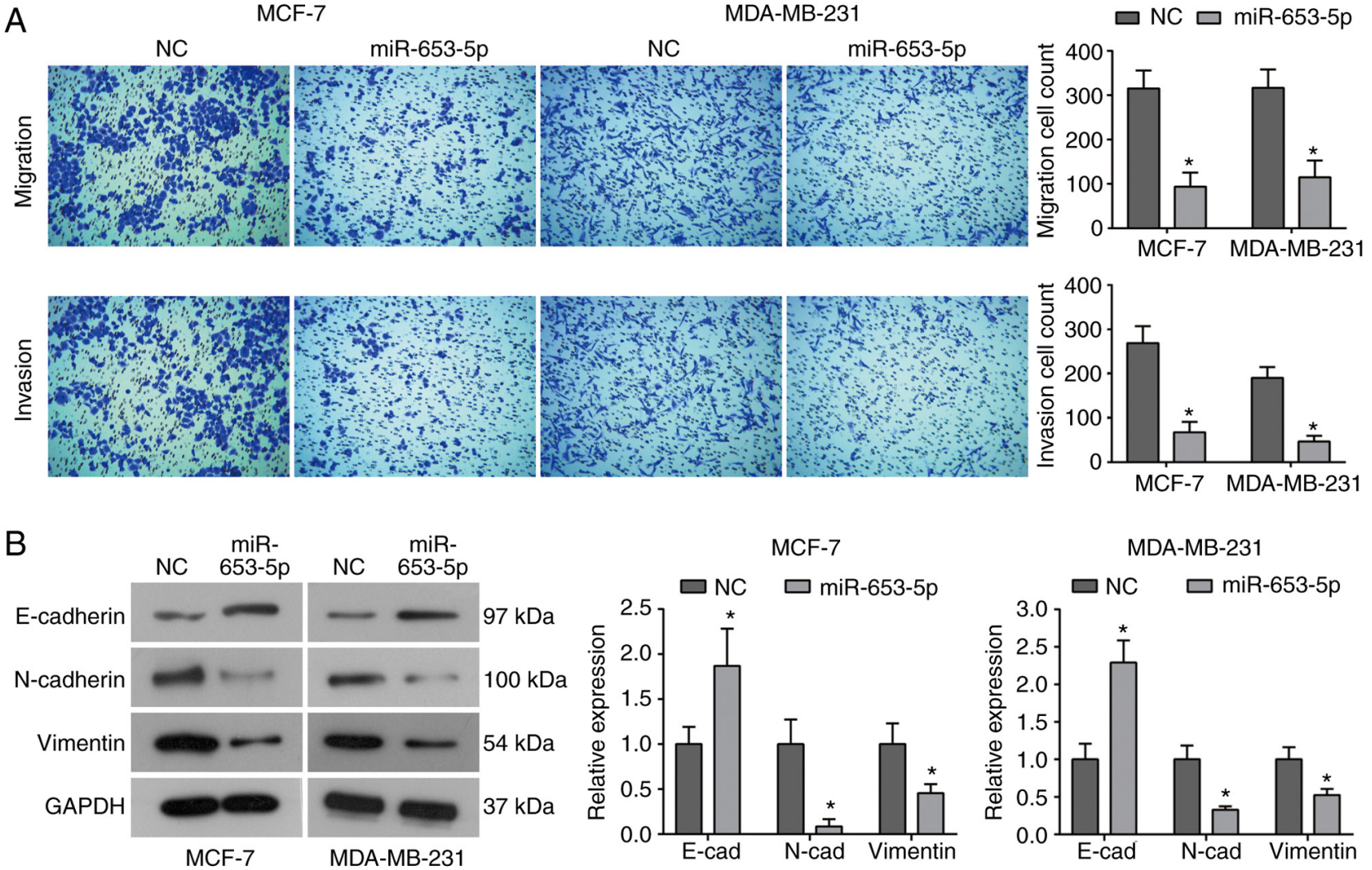

Figure 2. miR-653-5p suppresses cell migration, invasion and EMT in breast cancer cells. (A) Following transfection for $24 \mathrm{~h}$, cell migration and invasion were examined using a Transwell assay. Magnification, x100. (B) Western blotting was used to determine the relative expression of EMT-related proteins in MCF-7 and MDA-MB-231 cells transfected with miR-653-5p mimics. Experiments were performed in triplicate. "P<0.05 vs. NC. NC, negative control; miR, microRNA; EMT, epithelial-mesenchymal transition.

the expression of anti-apoptotic protein Bcl-2, and upregulated the expression of pro-apoptotic proteins, Bax and cleaved caspase-9, in both MCF-7 and MDA-MB-231 cells (Fig. 3B). Therefore, indicating that miR-653-5p promotes apoptosis in breast cancer cells, possibly by regulating the Bcl-2/Bax axis and activating caspase- 9 .

It is commonly known that the Akt/mTOR signaling pathway plays a critical role in regulating cell proliferation, differentiation, invasion and survival, as well as tumorigenesis and progression (17). Thus, the effect of miR-653-5p on the activation of the Akt/mTOR signaling pathway was evaluated in breast cancer cells. As determined by western blotting, it was found that miR-653-5p overexpression did not affect the total expression of Akt and mTOR, but it did significantly reduce the phosphorylation of Akt and mTOR in both MCF-7 and MDA-MB-231 cells (Fig. 3C), suggesting that miR-653-5p inhibits activation of the Akt/mTOR signaling pathway in breast cancer cells. These results indicated that the Akt/mTOR signaling pathway may be implicated in the suppressive role of miR-653-5p in breast cancer cells.

miR-653-5p suppresses the proliferation and migration of breast cancer cells by targeting MAPK6. To further elucidate the molecular mechanism by which miR-653-5p inhibits breast cancer cell proliferation and migration, the target gene of miR-653-5p was identified by bioinformatics analysis and a dual-luciferase reporter assay. It was predicated by bioinformatics analysis (TargetScan and StarBase) that the 3'UTR of MAPK6 mRNA contained a potential binding site for miR-653-5p (Fig. 4A). The dual-luciferase reporter assay demonstrated that miR-653-5p mimics resulted in a significant decrease in luciferase activity of the wt 3'UTR of MAPK6, but the luciferase activity of the mut 3'UTR of MAPK6 was not affected by miR-653-5p mimics (Fig. 4B). Moreover, western blotting further confirmed that miR-653-5p overexpression significantly decreased the expression of MAPK6 in both MCF-7 and MDA-MB-231 cells (Fig. 4C). MCF-7 and MDA-MB-231 cells were transfected with pcDNA3.1-MAPK6 to upregulate its expression (Fig. S1). Furthermore, upregulation of MPAK6 was able to partially reverse the miR-653-5p-induced inhibition of cell viability in both MCF-7 and MDA-MB-231 cells (Fig. 4D and E). Consistently, the decrease in cell migration caused by miR-653-5p was also reversed by MAPK6 overexpression in breast cancer cells (Fig. 4F). Collectively, these data support the hypothesis that miR-653-5p functions as a tumor suppressor in breast cancer by targeting MAPK6.

\section{Discussion}

It has been reported that miRNAs participate in regulating the expression of $1 / 3$ of human genes $(18,19)$. A growing number of studies have revealed that miRNAs are involved in the occurrence and development of cancers, and function 

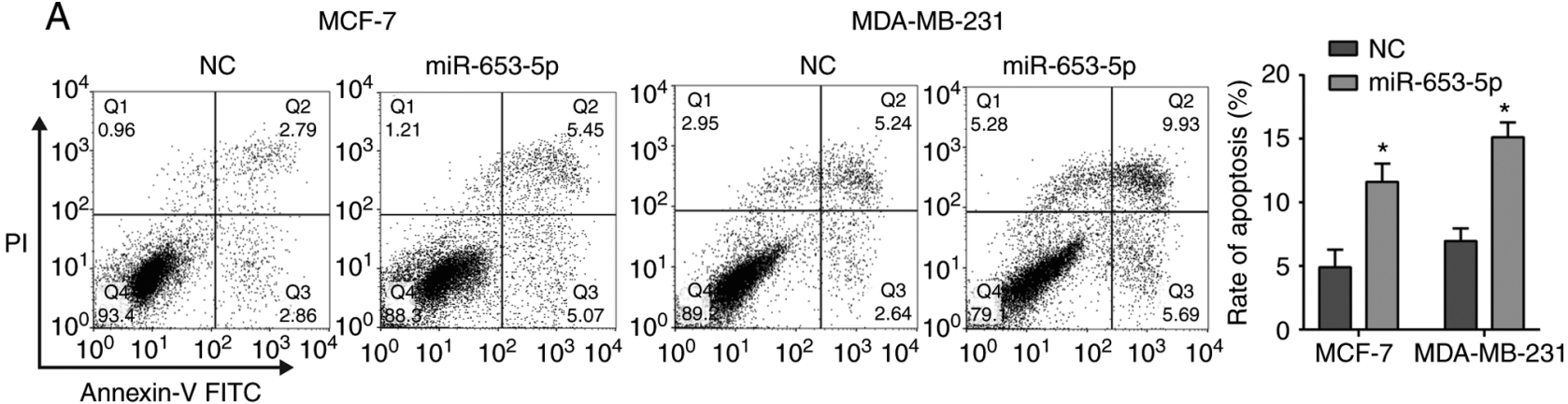

Annexin-V FITC
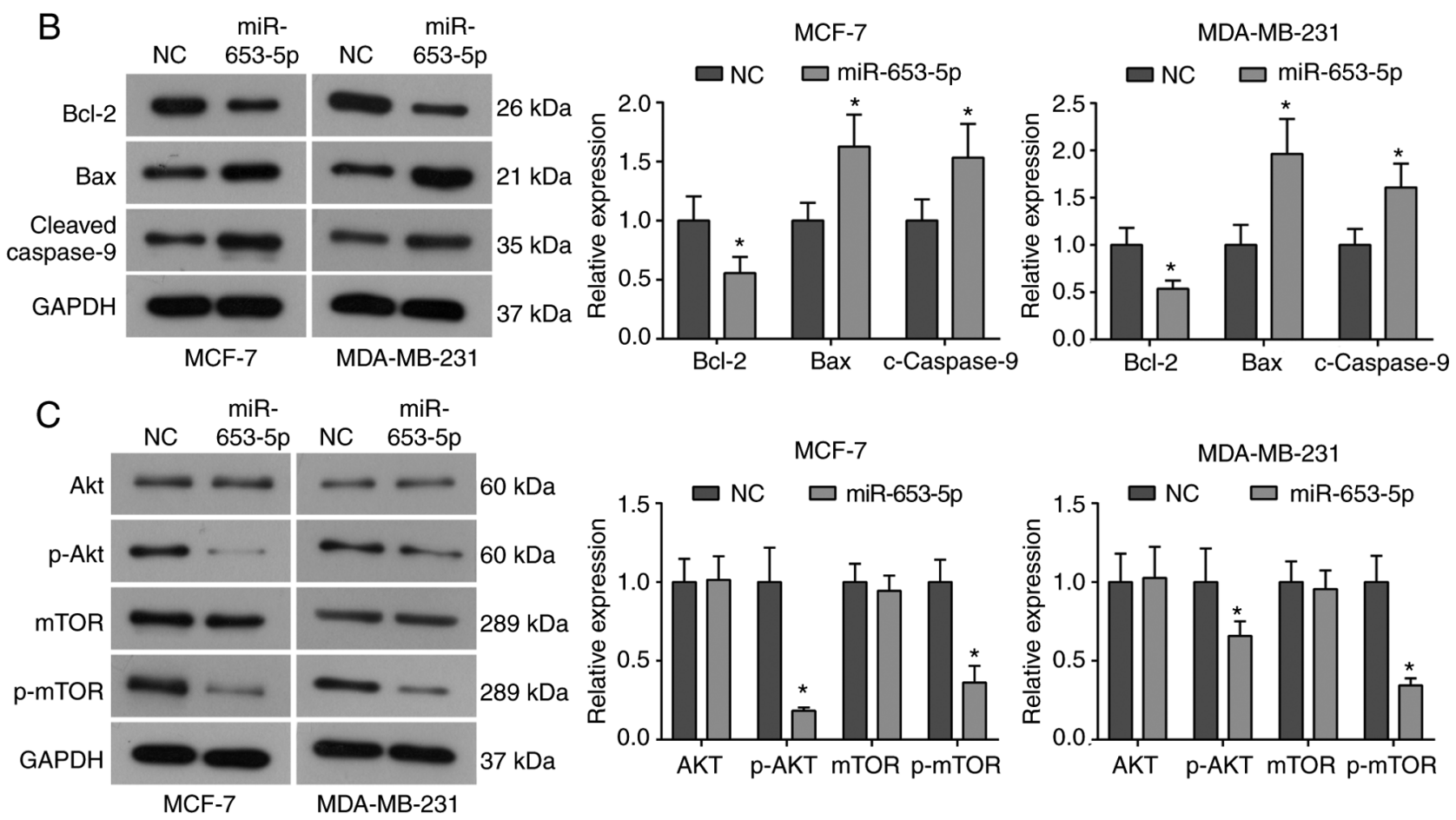

Figure 3. miR-653-5p promotes apoptosis and inhibits the Akt/mTOR signaling pathway in breast cancer cells. (A) Apoptosis in MCF-7 and MDA-MB-231 cells transfected with miR-653-5p mimics was determined using a flow cytometry assay. (B) Western blotting was used to determine the relative expression of apoptosis-related proteins. (C) Effect of miR-653-5p mimics on the Akt/mTOR signaling pathway was assessed using western blotting. Experiments were performed in triplicate. " $\mathrm{P}<0.05$ vs. NC. NC, negative control; miR, microRNA; mTOR, mammalian target of rapamycin; c-, cleaved; p-, phosphorylated.

as targets for cancer diagnosis and treatment (11-13). As a miRNA, miR-653 has been reported to have roles in cell proliferation, differentiation and apoptosis, which has been demonstrated in non-small cell lung cancer cells $(14,15)$. In the present study, it was found that miR-653-5p mimics significantly inhibited breast cancer cell proliferation, migration and invasion. Moreover, upregulation of miR-653-5p increased the expression of E-cadherin and decreased the expression of $\mathrm{N}$-cadherin and vimentin, suggesting that miR-653-5p suppresses EMT in breast cancer cells. Additionally, the results indicated that miR-653-5p promoted apoptosis by regulating $\mathrm{Bcl}-2 / \mathrm{Bax}$ and cleaved caspase- 9 . Collectively, these data suggested that miR-653-5p functions as a tumor suppressor in the growth and metastasis of breast cancer. However, there are limitations of the present study. For example, whether miR-653-5p-induced apoptosis is associated with its effect on cell migration is unknown, which will be further explored in a future study. Additionally, how miR-653-5p affects the expression of EMT-related proteins will be further studied.
As a well-known signaling pathway, Akt/mTOR signaling plays a key role in regulating various cellular functions, including cancer cell growth, metastasis and survival (17). It has been revealed that the Akt/mTOR signaling pathway is frequently overactivated in cancers (17). As a result, targeting the Akt/mTOR signaling pathway is considered to be an effective approach to control the growth and metastasis of tumors $(20,21)$. The present study found that miR-653-5p mimics inhibited the activation of the Akt/mTOR signaling pathway by decreasing the levels of phosphorylated (p)-Akt and p-mTOR in breast cancer cells. Therefore, miR-653-5p may be a potential therapeutic target for breast cancer treatment.

MAPK6, also known as ERK3, is an atypical MAPK. The MAPK6 pathway has been reported to be involved in inflammatory responses, and cell growth and differentiation (22). In addition, as an important downstream factor of MPAK6, p21 protein activated kinase (PAK) has been identified, and plays a key role in regulating cell adhesion and migration by regulating actin cytoskeleton dynamics (23). Therefore, MAPK6 plays an 
A

hsa-miR-653-5p 3' CCAAGUCAUCUCUAACAAAGUU 5'<smiles></smiles>

MAPK6 3' UTR (wt) 5'...CAGUCUCUUAGAAUUUGUUUCAU...3'

MAPK6 3' UTR (mut) 5'...CAGUCUCUUAGAAUUACAAAGUU...3'
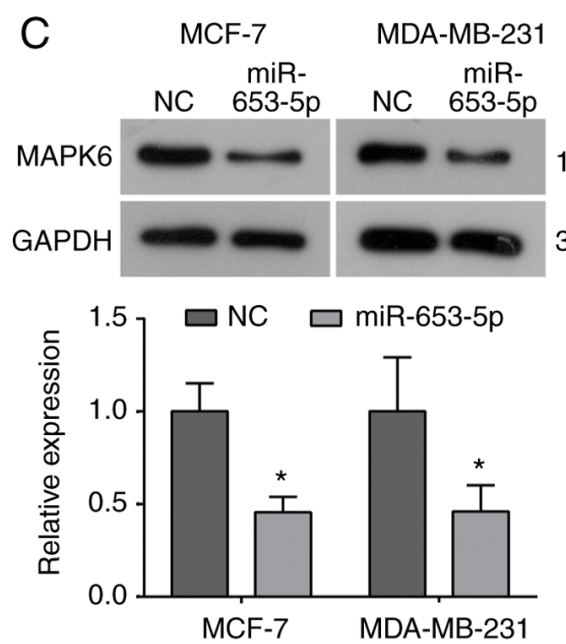

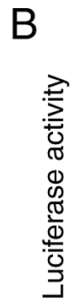

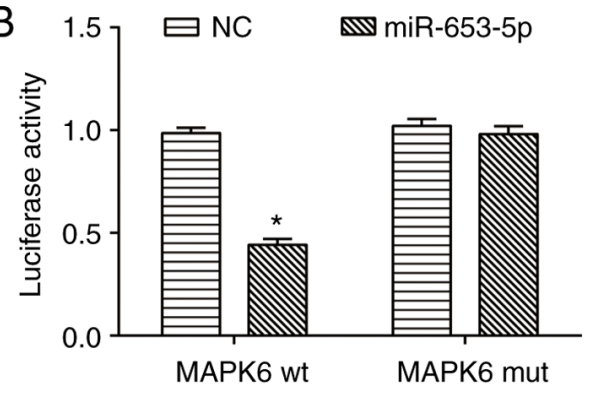

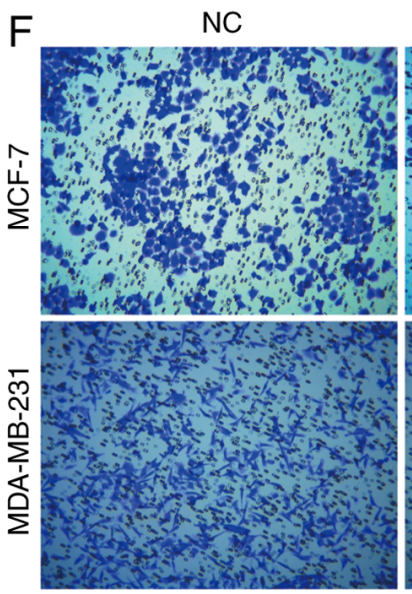

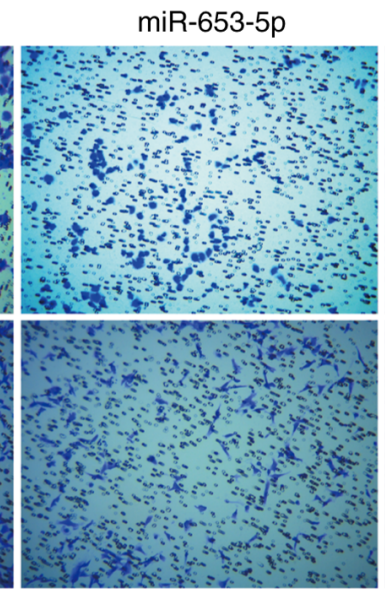

miR-653-5p+MAPK6

MCF-7

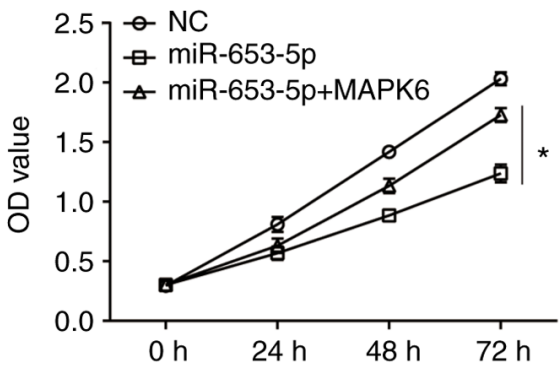

E

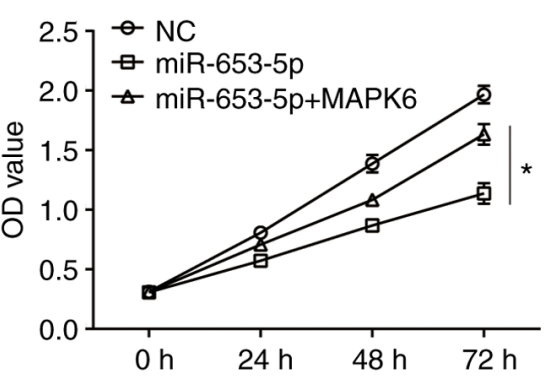

Figure 4. miR-653-5p inhibits cell proliferation and migration in breast cancer cells by targeting MAPK6. (A) Predicted binding site of miR-653-5p in 3'UTR of MAPK6 gene. (B) Effects of miR-653-5p on the luciferase activity of the MAPK6 3'UTR-wt/-mut. (C) Effect of miR-653-5p mimics on the expression of MAPK6 protein in breast cancer cells. (D) MCF-7 and (E) MDA-MB-231 cells were co-transfected with miR-653-5p mimics and pcDNA3.1-MAPK6 or transfected with miR-653-5p mimics alone, and cell viability was examined using a Cell Counting Kit-8 assay. (F) Cell migration was assessed using a Transwell assay. Magnification, $x 100$. Experiments were performed in triplicate. ${ }^{*} \mathrm{P}<0.05$ vs. NC or as indicated. NC, negative control; miR, microRNA; MAPK, mitogen-activated protein kinase 6; 3'UTR, 3'untranslated region; wt, wild-type; mut, mutant.

important role in cell migration. Furthermore, previous studies have demonstrated that MAPK6 is involved in the metastasis of cancer cells (24-26). MAPK6 has also been revealed to be involved in cell proliferation and apoptosis in laryngeal carcinoma as a target of miR-138 (27). MAPK6 knockdown inhibits the proliferation, migration and invasion of cervical cancer (28). Lv et al (29), reported that MAPK6 is upregulated in breast cancer and is associated with poor prognosis of patients. In the present study, MAPK6 was identified as a target of miR-653-5p that could be negatively regulated by miR-653-5p in breast cancer cells. Furthermore, upregulation of MAPK6 was able to partially reverse the inhibitory effects of miR-653-5p on the proliferation and migration of breast cancer cells. Taken together, these results indicated that miR-653-5p inhibits the progression of breast cancer by targeting MAPK6. Potapova et al (30) previously reported that MAPK9-induced apoptosis is highly dependent on p53 in MCF7 and other p53 wt cell lines, except for MDA-MB-231 cells with a p53 mutation. Previous studies have revealed that MAPK6, as a target gene of miRNAs, is involved in the regulation of cell apoptosis $(31,32)$. At present, we have not found any reports concerning MAPK6 and p53. We will further explore the specific underlying mechanism of MAPK6 in apoptosis and whether it is related to p53 in the future.

In summary, the present findings support the hypothesis that miR-653-5p acts as a novel tumor suppressor in the progression of breast cancer by directly targeting MAPK6. Consequently, the present study indicates that miR-653-5p may act as a novel target for the treatment of breast cancer. 


\section{Acknowledgements}

Not applicable.

\section{Funding}

No funding was received.

\section{Availability of data and materials}

The datasets used and/or analyzed during the current study are available from the corresponding author on reasonable request.

\section{Authors' contributions}

MZ designed the study and drafted the manuscript. MZ, HW and $\mathrm{XZ}$ performed the experiments. HW and FL performed the statistical analysis. All authors read and approved the final manuscript.

\section{Ethics approval and consent to participate}

Not applicable.

\section{Patient consent for publication}

Not applicable.

\section{Competing interests}

The authors declare that they have no competing interests.

\section{References}

1. Bray F, Ferlay J, Soerjomataram I, Siegel RL, Torre LA and Jemal A: Global cancer statistics 2018: GLOBOCAN estimates of incidence and mortality worldwide for 36 cancers in 185 countries. CA Cancer J Clin 68: 394-424, 2018.

2. Israel BB, Tilghman SL, Parker-Lemieux K and Payton-Stewart F: Phytochemicals: Current strategies for treating breast cancer. Oncol Lett 15: 7471-7478, 2018.

3. Calaf GM and Roy D: Metastatic genes targeted by an antioxidant in an established radiation- and estrogen-breast cancer model. Int J Oncol 51: 1590-1600, 2017.

4. Cristofanilli M and Fortina P: Circulating tumor DNA to monitor metastatic breast cancer. N Engl J Med 369: 93, 2013.

5. Si W, Shen J, Du C, Chen D, Gu X, Li C, Yao M, Pan J, Cheng J, Jiang D, et al: A miR-20a/MAPK1/c-Myc regulatory feedback loop regulates breast carcinogenesis and chemoresistance. Cell Death Differ 25: 406-420, 2018.

6. Chen S, Wang Y, Zhang JH, Xia QJ, Sun Q, Li ZK, Zhang JG, Tang MS and Dong MS: Long non-coding RNA PTENP1 inhibits proliferation and migration of breast cancer cells via AKT and MAPK signaling pathways. Oncol Lett 14: 4659-4662, 2017.

7. Fabbri M: TLRs as miRNA receptors. Cancer Res 72: 6333-6337, 2012.

8. Torres S, García-Palmero I, Bartolomé RA, Fernandez-Aceñero MJ, Molina E, Calviño E, Segura MF and Casal JI: Combined miRNA profiling and proteomics demonstrates that different miRNAs target a common set of proteins to promote colorectal cancer metastasis. J Pathol 242: 39-51, 2017.

9. Garzon R, Marcucci G and Croce CM: Targeting microRNAs in cancer: Rationale, strategies and challenges. Nat Rev Drug Discov 9: 775-789, 2010.

10. Rupaimoole R and Slack FJ: MicroRNA therapeutics: Towards a new era for the management of cancer and other diseases. Nat Rev Drug Discov 16: 203-221, 2017.
11. Liu X, Bi L, Wang Q, Wen M, Li C, Ren Y, Jiao Q, Mao JH, Wang C, Wei G and Wang Y: miR-1204 targets VDR to promotes epithelial-mesenchymal transition and metastasis in breast cancer. Oncogene 37: 3426-3439, 2018.

12. El Helou R, Pinna G, Cabaud O, Wicinski J, Bhajun R, Guyon L, Rioualen C, Finetti P, Gros A, Mari B, et al: miR-600 acts as a bimodal switch that regulates breast cancer stem cell fate through WNT signaling. Cell Rep 18: 2256-2268, 2017.

13. Liu W, Xu Y, Guan $\mathrm{H}$ and Meng H: Clinical potential of miR-940 as a diagnostic and prognostic biomarker in breast cancer patients. Cancer Biomark 22: 487-493, 2018.

14. Cao YL, Dong W, Li YZ and Han W: MicroRNA-653 inhibits thymocyte proliferation and induces thymocyte apoptosis in mice with autoimmune myasthenia gravis by downregulating TRIM9. Neuroimmunomodulation 26: 7-18, 2019.

15. Yuan L, Feng Y, Li L, et al: Effect of microRNA-653 on biological characteristics of human non-small cell lung cancer cells by targeting OIP5 gene and regulating mTOR signaling pathway. J Mod Oncol 26: 831-837, 2018.

16. Livak KJ and Schmittgen TD: Analysis of relative gene expression data using real-time quantitative PCR and the 2(-Delta Delta C(T)) method. Methods 25: 402-408, 2001.

17. Tokunaga E, Oki E, Egashira A, Sadanaga N, Morita M, Kakeji Y and Maehara Y: Deregulation of the Akt pathway in human cancer. Curr Cancer Drug Targets 8: 27-36, 2008.

18. Paladini L, Fabris L, Bottai G, Raschioni C, Calin GA and Santarpia L: Targeting microRNAs as key modulators of tumor immune response. J Exp Clin Cancer Res 35: 103, 2016.

19. Rupaimoole R, Calin GA, Lopez-Berestein G and Sood AK: miRNA deregulation in cancer cells and the tumor microenvironment. Cancer Discov 6: 235-246, 2016.

20. Morgensztern D and McLeod HL: PI3K/Akt/mTOR pathway as a target for cancer therapy. Anticancer Drugs 16: 797-803, 2005.

21. Mayer IA and Arteaga CL: The PI3K/AKT pathway as a target for cancer treatment. Annu Rev Med 67: 11-28, 2016.

22. Brand F, Schumacher S, Kant S, Menon MB, Simon R, Turgeon B, Britsch S, Meloche S, Gaestel M and Kotlyarov A: The extracellular signal-regulated kinase 3 [mitogen-activated protein kinase 6 (MAPK6)]-MAPK-activated protein kinase 5 signaling complex regulates septin function and dendrite morphology. Mol Cell Biol 32: 2467-2478, 2012.

23. Al-Mahdi R, Babteen N, Thillai K, Holt M, Johansen B, Wetting HL, Seternes OM and Wells CM: A novel role for atypical MAPK kinase ERK3 in regulating breast cancer cell morphology and migration. Cell Adh Migr 9: 483-494, 2015.

24. Evtimova V, Schwirzke M, Tarbé N, Burtscher H, Jarsch M, Kaul S and Weidle UH: Identification of breast cancer metastasis-associated genes by chip technology. Anticancer Res 21: 3799-3806, 2001

25. Liang B, Wang S, Zhu XG, Yu YX, Cui ZR and Yu YZ: Increased expression of mitogen-activated protein kinase and its upstream regulating signal in human gastric cancer. World J Gastroenterol 11: 623-628, 2005.

26. Long W, Foulds CE, Qin J, Liu J, Ding C, Lonard DM, Solis LM, Wistuba II, Qin J, Tsai SY, et al: ERK3 signals through SRC-3 coactivator to promote human lung cancer cell invasion. J Clin Invest 122: 1869-1880, 2012.

27. Zhou YH, Huang YY and Ma M: MicroRNA-138 inhibits proliferation and induces apoptosis of laryngeal carcinoma via targeting MAPK6. Eur Rev Med Pharmacol Sci 22: 5569-5575, 2018.

28. Wu J, Zhao Y, Li F and Qiao B: MiR-144-3p: A novel tumor suppressor targeting MAPK6 in cervical cancer. J Physiol Biochem 75: 143-152, 2019.

29. Lv P, Qiu X, Gu Y, Yang X, Xu X and Yang Y: Long non-coding RNA SNHG6 enhances cell proliferation, migration and invasion by regulating miR-26a-5p/MAPK6 in breast cancer. Biomed Pharmacother 110: 294-301, 2019.

30. Potapova O, Gorospe M, Dougherty RH, Dean NM, Gaarde WA and Holbrook NJ: Inhibition of c-Jun N-terminal kinase 2 expression suppresses growth and induces apoptosis of human tumor cells in a p53-dependent manner. Mol Cell Biol 20: 1713-1722, 2000.

31. Hu C, Huang S, Wu F and Ding H: miR-98 inhibits cell proliferation and induces cell apoptosis by targeting MAPK6 in HUVECs. Exp Ther Med 15: 2755-2760, 2018.

32. Hao W, Zhao ZH, Meng QT, Tie ME, Lei SQ and Xia ZY: Propofol protects against hepatic ischemia/reperfusion injury via miR-133a-5p regulating the expression of MAPK6. Cell Biol Int 41: 495-504, 2017.

This work is licensed under a Creative Commons Attribution-NonCommercial-NoDerivatives 4.0 International (CC BY-NC-ND 4.0) License. 